\title{
Productive and Security in Remote Sensor Network using SNR
}

\author{
Sk. Shama, Yashwanth Inuganti, M. B. Bhavana, A. Jagapathi
}

\begin{abstract}
Remote Sensor Networks is presently generally spread the nation over. This contraption faculties and screens the physical state of our condition and assembles every one of the information gathered at a focal area called sink. The contribution to this contraption is the physical conditions and changes over it into electrical sign A collection of spatially distributed and dedicated sensors als o known as wire less sensor network, the hubs in the system have a restricted transmission, less vitality and less space. So to improve this we use grouping methods to give an effective method to build the lifetime of a WSN hence we are utilizing Signal to Noise Ratio (SNR) based bunching instrument This plan proposes an Efficient and Secure Routing Protocol for Wireless Sensor Networks through SNR-based Unique Bunchin $g$ (ESRPSDC) that can isolate the sensor hubs into a few meeting $s$ called as groups where one hub will be group head $(\mathrm{CH})$ and no n-cluster head (NCH) adherents. These supporters will detect and gather the information and the information will be sent to group head. The bunch head will at that point send the information to the sink. We need group heads on the grounds that the hubs can't straightforwardly send information to the sink in view of less vitality.

Keywords:Cluster Head, Wireless Sensor Networks, Noise Rat
\end{abstract} io signal

\section{INTRODUCTION}

Remote Sensor Networks are structured or unstructured system. WSN are unstructured systems as they don't have a fixed topology on the grounds that the hubs continue changing the areas persistently. WSN can be static or dynamic grouping. Here we utilize dynamic grouping instrument in light of the fact that the designs will be done consequently by utilizing dynamic directing conventions. Therefore, during runtime, the hubs can join and leave a corr espondence framework which is a troubling errand to orches trate them in a correspondence framework. So to take care of this sort of issues bunching calculations are proposed to give an organized method for correspondence for an unstructured system. In bunching calculation, the sensor hubs are separated into a few groups by picking their group heads which performs information total and sends the information to the base station in this way spare the vitality of different groups. Grouping is the movement which isolates a lot of comparable items.

Revised Manuscript Received on December 30, 2019.

* Correspondence Author

Sk. Shama, Assistant Professor Department of Computer Science and Engineering, Koneru Lakshmaiah Education Foundation situated, Vaddeswarm, India.

Yashwanth Inuganti, Department of CSE Bachelor of Technology, Koneru Lakshmiah Educational Foundation, Andhra Pradesh, India.

M. B. Bhavana, Department of CSE Koneru Lakshmiah Educational Foundation, Andhra Pradesh, India.

A. Jagapathi, Department of CSE Bachelor of Technology, Koneru Lakshmiah Educational Foundation, Andhra Pradesh, India.

(C) The Authors. Published by Blue Eyes Intelligence Engineering and Sciences Publication (BEIESP). This is an open access article under the CC BY-NC-ND license (http://creativecommons.org/licenses/by-nc-nd/4.0/)
The centers in clustered remote sensor frameworks are of two sorts one is the gathering head which does data aggregation and talks with other pack heads present in the framework to move the data to the base station and the other is the disciples which identifies the physical conditions and change yield data to their individual bundle heads. In this paper, we are utilizing SNR gathering strategy by which the lifetime of a battery gets expanded just as security is additionally given. Security is a significant criterion in this in light of the fact that with no security parcel of information can be lost which we can't discover or which we couldn't recoup. We are having many different algorithms but those algorithms may not have the option to provide security to the hubs in a sensor network. This method will have four different stages which we have described in the paper. SNR means signal to noise ratio by knowing the value of SNR we can find whether the hub is affected or not. If we find a specific hub then we will not send any message to that hub.

\section{TYPES OF CLUSTERING ALGORITHMS IN WIRELESS SENSORS}

\section{K-Means Clustering}

It is a disseminated grouping calculation [1]. In this clustering mechanism there are two ways to find out determination criteria for bunch head they are: -

1. Euclidian separations

2. Remaining energies of hubs

Each center point transmits the data to a nearby center which stores this information in a once-over. After it has accumulated the data from its centers it plays out the $\mathrm{k}$-implies gathering estimation. Occasion to sink coordinated bunching

It gives high capability to the extent essentialness usage. Exactly when a center point finds an event, it sends its reports to base station. A sensor center sends the assembled data to pack head thusly avoiding reiteration [2].

This framework gives new redesigns:

1. Clustering is conceivably done when an event occurs, so no trivial packing modifies ought to be performed.

2. There is least improvement of data in the gathering since packs are structure toward the way from event to base station. 3. Cluster heads are looked over up-stream centers and non-bunch centers are browsed downstream center points. So stream of data is practically uni-directional.

\section{Load balanced clustering Scheme}

The entire errands of a system is finished by bunch head. because of substantial burden bunch head will murder it by greedy all of its vitality.

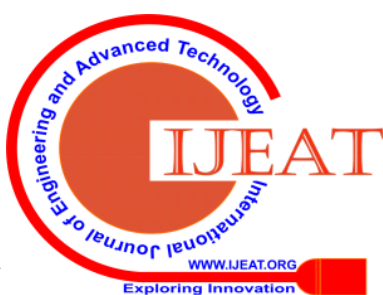


In load adjusted bunching [4] conspire Associate in Nursing associate hub is chosen to modify the cluster to form a path for play out its information accumulation and knowledge handling task. Collaborator hub transmits the data to base station. Bunch head method the got data and sends it to collaborator hub. mitt hub sends this data to base station.

\section{Hybrid vitality Efficient Distributed Clustering}

It considers 2 components to create a hub cluster head or not. These are as per the following:

1. Residual vitality

2. Intra-bunch correspondence price.

All the cluster heads within the system gets systematically disseminated, thus potency of network decreases and typically elects additional cluster heads.

\section{Less vitality Adaptive Clustering}

The principle purpose of this calculation is that every hub got to get a chance to become cluster head. The heap is equally spread among each one of the hubs gift in system.

\section{Energy Efficient Hierarchical Clustering}

In this [3], each hub chooses to become bunch or not. On the off likelihood that it becomes cluster head, at that time it publicizes to any or all hubs that the individual hub is bunch head. Any node that receives this message can become a member of that cluster head.

\section{Weight Based Clustering Protocol}

In this [3], every hub chooses to become bunch or not. On the off chance that it becomes cluster head, at that point it publicizes to any or all hubs that the individual hub is bunch head. Any node that receives this message will become a member of the Weight id is employed to decide on a Cluster Head. This weight [5] area unit typically numerable as leftover vitality and separation of hubs from cluster head or vary of times a hub becoming bunch head smitten by the calculation. Bunches area unit shaped therefore base vitality is employed.

The principle purpose of this may be to:

1. To increment the period of sensor hubs by choosing bunch head that has high leftover vitality.

2. To evade the appointment of low vitality sensor hubs as cluster heads.

In this paper we've got an inclination to utilize economical and Secure Routing Protocol through SNR primarily based powerful grouping which may give every proficiency of sensors and steering vogue dependent on security system. ESRPSDC abuses Energy efficiency Level primarily based clump Protocol [6] and security techniques area unit as determined in [7] at cluster head.

\section{PROPOSED METHODOLOGY}

\subsection{SNR BASED DYNAMIC CLUSTERING}

Here at first all hubs are heterogeneous hubs with restricted inventory of vitality. The procedure is separated into four unique stages: -

\section{Instatement}

The sink communicates a Request message to all the hubs and will be separated into groups dependent on hubs more like each other and afterward bunch Id will be framed and a group table.

\subsection{FLOW CHART}

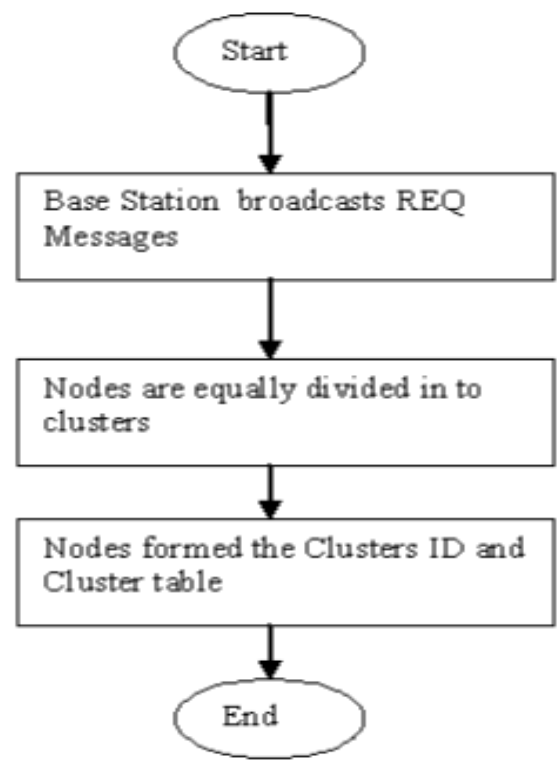

Fig 1: Instatement Phase

\section{Bunch Head choice dependent on vitality}

In this highest energy hub will be picked as group head (CH) next highest elevated will be picked as next bunch head.

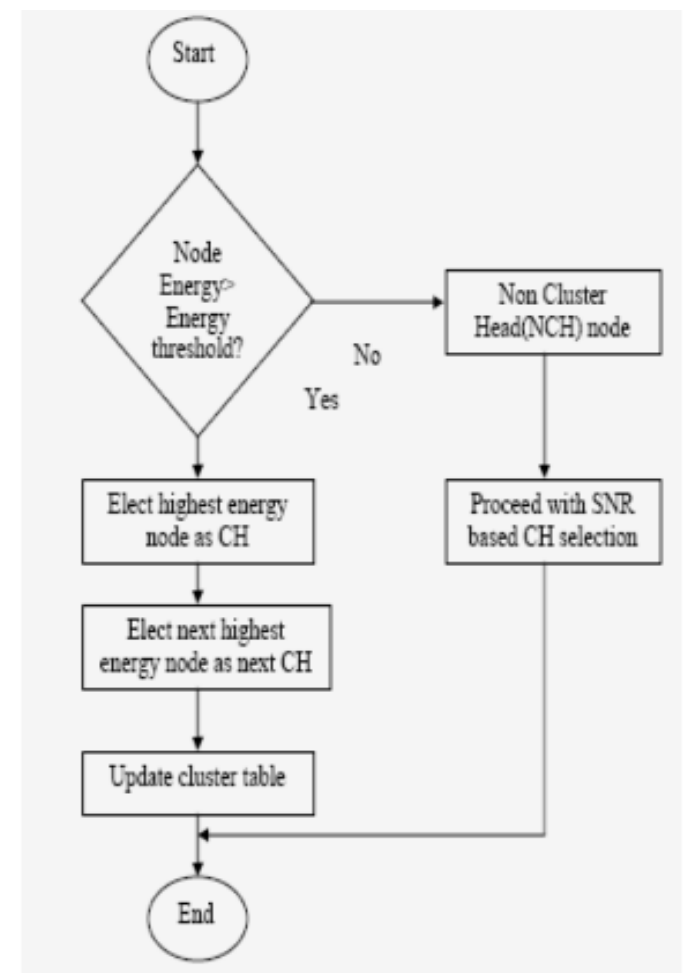

Fig 2: Vitality based CH choice 


\section{SNR based bunch head choice}

In this stage all hubs who are sent at arrange limits or who are not in scope of bunch head will pick substitute neighbors to transfer information to group head.

\section{Intruder Detection}

On this phase by using ID's base station will identify the attacker and inform to the sensors. Gatecrashers are not really situated at the middle [8] in a multi bounce sensor organize.

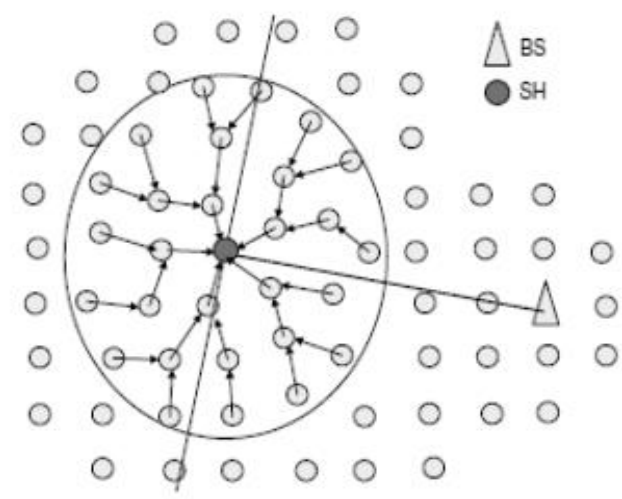

Fig 3: Affected area

\section{RESULT AND DISCUSSION}

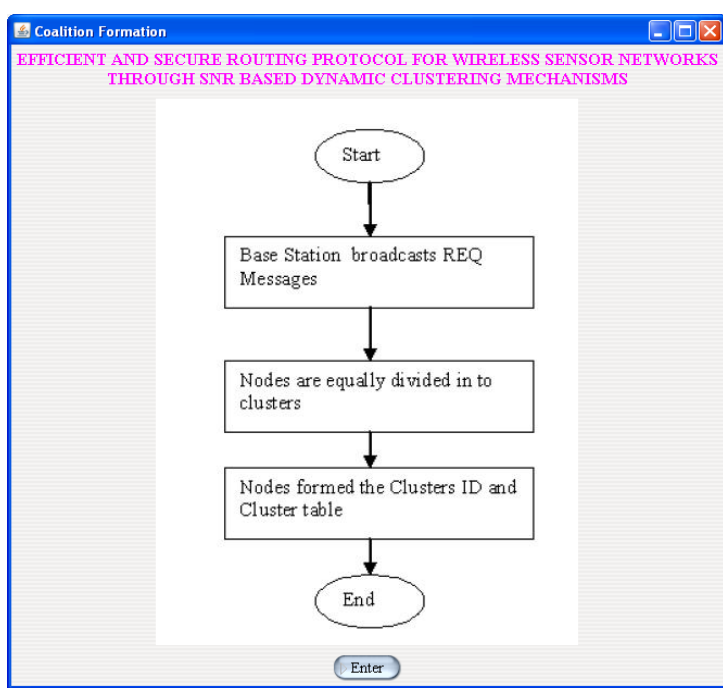

Fig 4: Click on enter to start with initialization

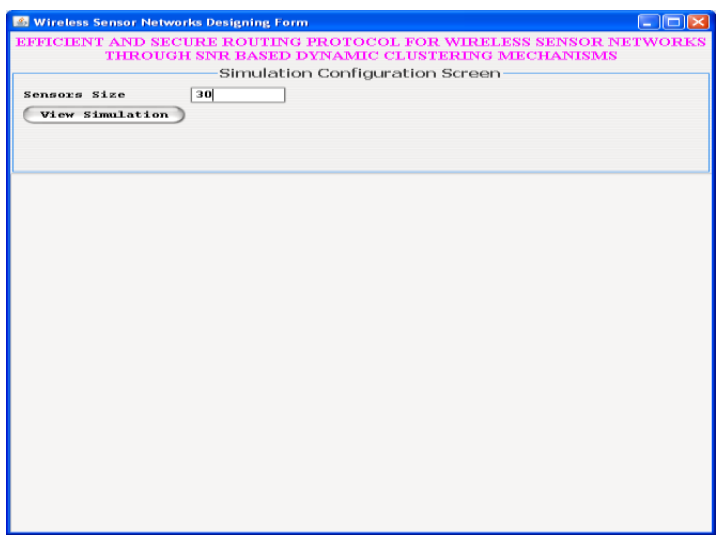

Fig 5: In the given screen enter the sensor size as30 whichmean there will be 30 sensor nodes in simulation panel.

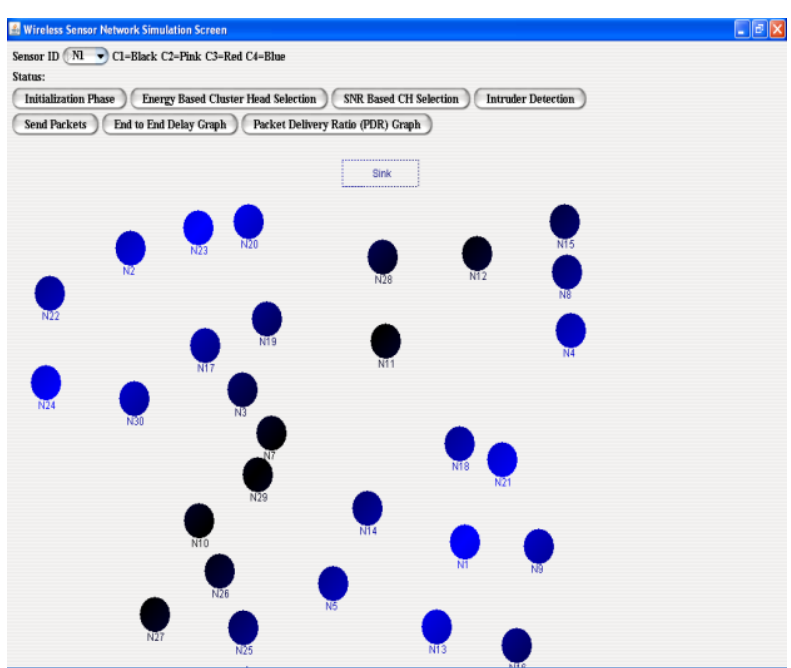

Fig 6: All 30 sensors are placed at random position. Now click on 'Initialization Phase' to form clusters.

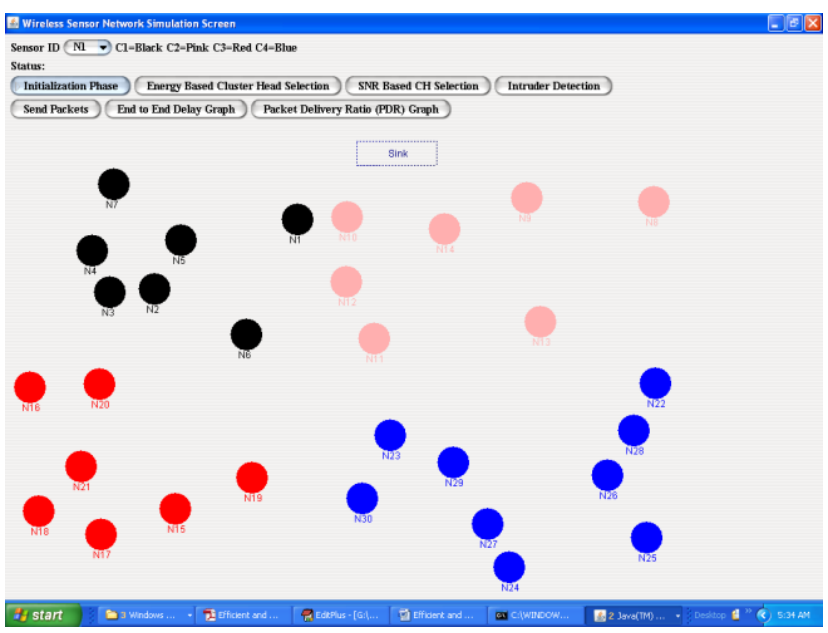

Fig 6: Four clusters are formed each with different colors.

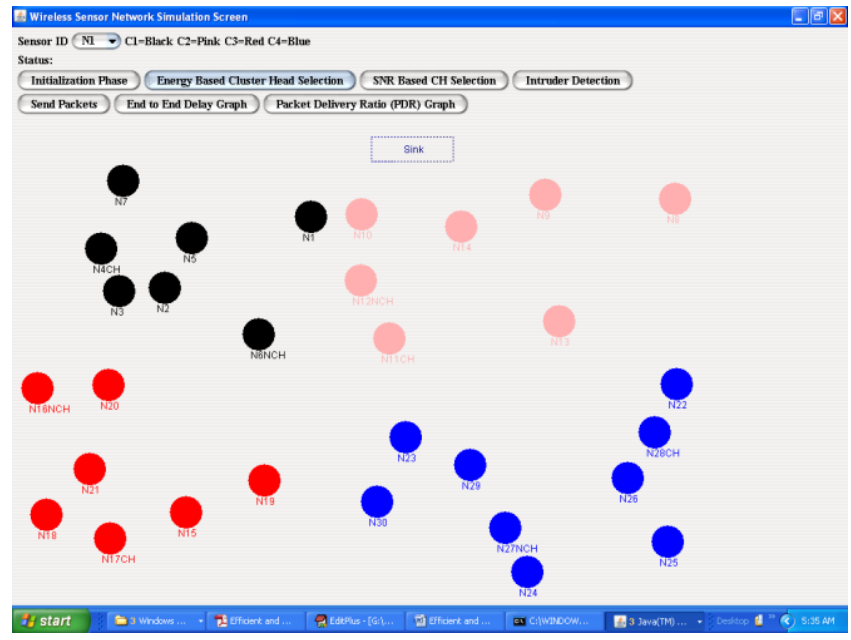

Fig 7: Selected cluster head is marked with name ' $\mathrm{CH}$ ' and 'NCH'. 


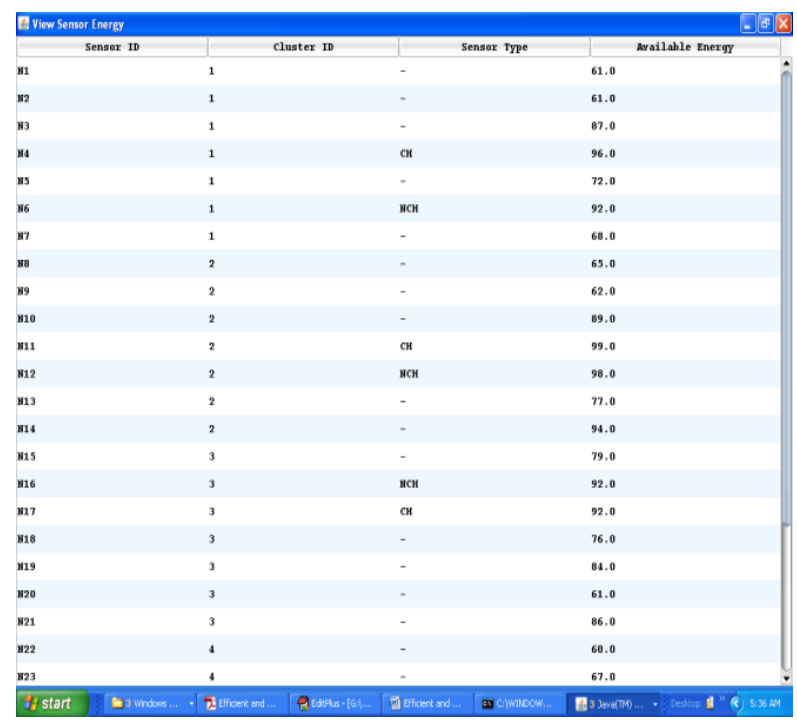

Fig 8: Sensor id and cluster id is seen with chosen cluster head with available energy in the last column. In each cluster we can see sensor with maximum battery is chosen as cluster head.

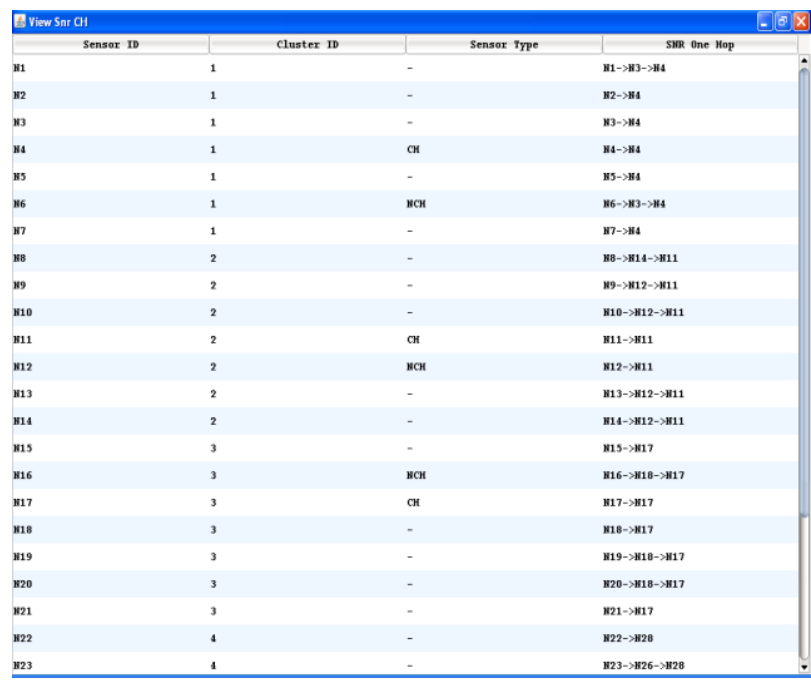

Fig 9: We can see N1 reach to N4 cluster head via neighbor N3, if sensor direct range in cluster head then it will not take any neighbor.

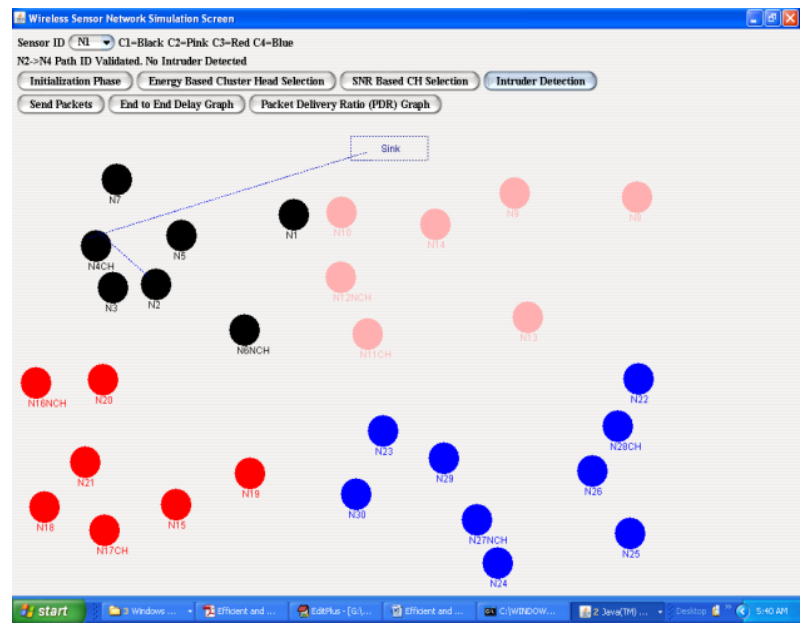

Fig 10: Base station send request to sensors via cluster head and if selected path contains no ID then the path contains intruder.

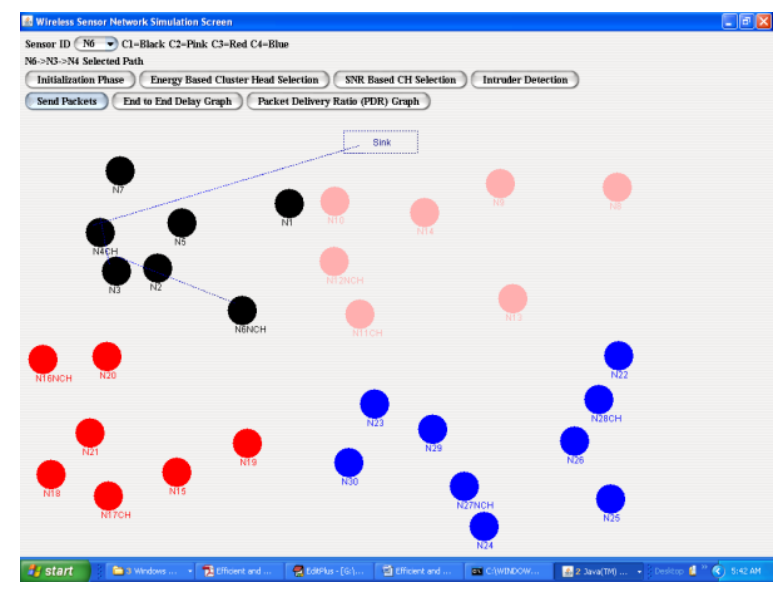

Fig 11: Packet is sending from N6 to sink.

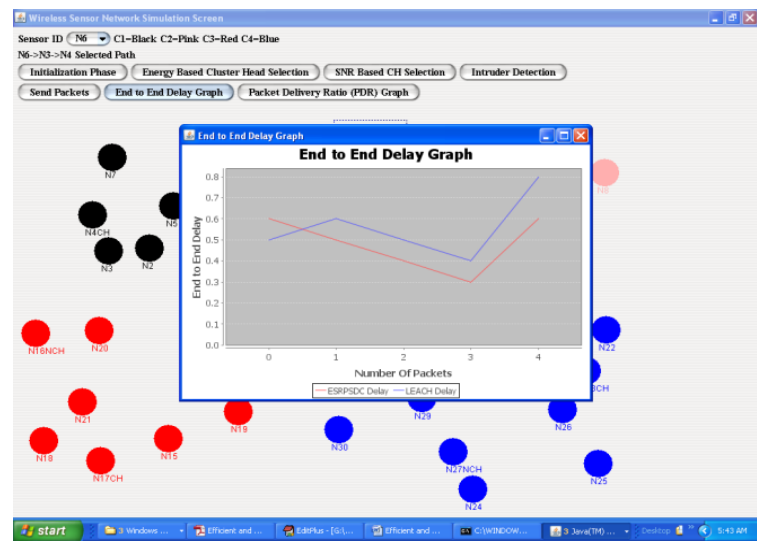

Fig 12: In above diagram $X$-hub speaks to number of parcels

and y-pivot speaks to bundle delay. Blue line is existing LEACH convention and filter will have high postponement as it won't choose bunch head on high vitality so system will shutdown prior. Red line alludes to propose work. Start to finish defer implies time consumed for delivery of information from one end to other.

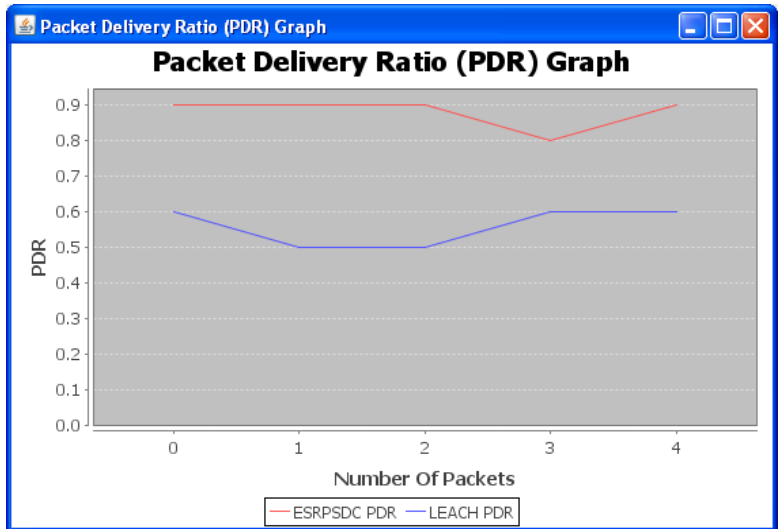

Fig 13: $x$-pivot speaks to number of parcels and $y$-hub speaks to PDR value. PDR is the proportion of effectively conveyed bundles to goal to those created by the CBR sources.

Published By:

Blue Eyes Intelligence Engineering

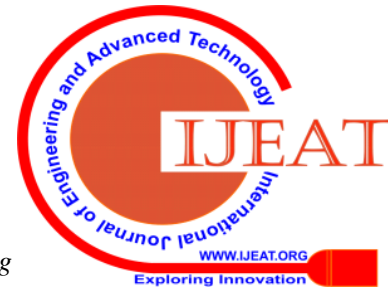




\section{CONCLUSION}

In this paper, directing conventions for vitality effective information assortment through SNR based powerful bunching have been proposed. We can see that [8] for solid vitality proficient correspondence we need to consider both separation and mistake pace of every hub. While picking a specific connection it ought to be guaranteed that it is sans mistake delivery. This is significant in multi bounce remote situations where bundle misfortune can be higher [9]. SNR means signal to noise ratio by knowing the value of SNR we can find whether the hub is affected or not. If we find a specific hub then we will not send any message to that hub. This concludes that ESRPSDC is more effective than LEACH algorithm as we can see in the graphs.

\section{REFERENCES}

1. S. Basagni, "Distributed cluster algorithmic program for AdHoc-Networks.," Proceedings of International conference Parallel Algorithms, Architecture and Network, pp.310-315, Gregorian calendar month 1999.

2. Alper Bereketli,Ozgur B. Akan (2009),Event-to- Sink Directed bunch in Wireless sensing element Networks ,Next generation Wireless Communications Laboratory (NWCL) Department of Electrical and natural philosophy Engineering Middle East Technical University, Ankara Turkey, 06531 978-1-4244-2948-6/09/\$25.00 ( IEEE.

3. N.Wakamiya,J.Kamimur, and M.Murata Energy - Efficient Clustering Method for Data Gathering in Sensor Networks, Proceedings of Workshop on Broadband Advanced Sensor Networks, Vol.103, pp. 31-36, April 2004.

4. LBCS: A Load Balanced Clustering Scheme in Wireless Sensor Networks, Shujuaan Jin, Keqiu Li (2009).Third International Conference on Multimedia and Ubiquitous Engineering, 978-07695-3658-3/09 \$25.00 ( ) 2009 IEEE DOI 10.1109/MUE.2009.47.

5. Biswanath Dey Ravi Tandon" Weight Based Clustering In Wireless Sensor Networks", 978-1-4673-5952-8/13/\$31.00 (c) IEEE. M.Diwakar and S.Kumar, - An Energy Efficient Level based Clustering routing protocol for WSN\| International Journal of Advanced Smart Senso Network Systems, Vol.2, issue 2, pp 55- 65, April 2012

6. R.Amutha and S.Ganesh Efficient and Secure Routing Protocol for Wireless Sensor Networks through Two level intrusion detection mechanismll Wulfenia Journal, Vol.19, pp.388- 406, December 2012.

7. N.Gupta and K.Kant - Application based Study on Wireless Sensor Network", International Journal of Computer Applications, Vol. 21, March 2011.

8. R.Amutha and S.Ganesh - Network Security in Wireless Sensor Networks Using Triple Umpiring Systemll European Journal of Scientific Research, Vol.64, issue 1, pp.128-145, June 2011.

9. R.Amutha and S.Ganesh Efficient and Secure Routing Protocol for Wireless Sensor Networks through Optimal Power Control and Optimal Handoff-Based Recovery Mechanismll Journal of Computer Networks and Communications, Vol.2012, 8 pages, July 2012.

10. D. K., \& Smys Anguraj, S. (2019). Trust-based intrusion detection and clustering approach for wireless body area networks. Wireless Personal Communications, 104(1) doi:10.1007/s11277-018-6005-x

11. [11]. Bhandari, R. R., \& Rajasekhar, K. (2016). A Study on improving the network life time maximization for wireless sensor network using cross layer approach. International Journal of Electrical and Computer Engineering, 6(6), 3080-3086. doi:10.11591/ijece.v6i6.11208

12. r, S., \& Srinivasu, Dongaonka N. (2016). Algorithms for energy efficiency \& coverage problems in wireless sensor networks. International Journal of Control Theory and Applications, 9(34), 497-501.

13. K. Raju ,\& G. S. N Kumar Naik, (2016). Synthesis of linear concentric ring arrays with high directivity and low sidelobe levels. Wireless Personal Communications, 87(1), 1-15 doi:10.1007/s11277-015-3021-y

14. Boddu, N., Vatambeti, R., \& Bobba, V. (2017). "Achieving energy efficiency and increasing the network life time in MANET through fault tolerant multi-path routing". International Journal of Intelligent $\begin{array}{llll}\text { Engineering and } & \text { Systems, } 166-172 .\end{array}$ doi:10.22266/ijies2017.0630.18

15. Buvanesvari, M., Uthayakumar, J., \& Amudhavel, J. (2017).” Fuzzy based clustering to maximize network lifetime in wireless mobile

sensor networks". Journal of Advanced Research in Dynamical and Control Systems, 9(Special Issue 12), 2133-2143.

16. Satyanarayana, Chowdary, K., \& S, K. V. V. (2017). Malicious node detection and reconstruction of network in sensor actor network. Journal of Theoretical and Applied Information Technology, 95(3), 582-591.

17. Shaik, R., \& Ahamad, S. S. (2017). "An agent-based hybrid approach for dynamic key management system in dynamic wireless sensor network". Journal of Advanced Research in Dynamical and Control Systems, 9(2), 213-227.

18. Shaik, R., \& Ahamad, S. S. (2017). "Enhanced attack resistant agent based dynamic key management in dynamic wireless sensor networks". International Journal of Civil Engineering and Technology, $8(12), 69-76$.

19. Somu, V., Kamesh, D. B. K., Sastry, J. K. R., \& Sitara, S. N. M. (2017). Snort rule detection for countering in network attacks doi:10.1007/978-981-10-3153-3_57.

20. T., \& Amudhavel, J., Vengattaraman Uthayakumar , (2017).” Data compression algorithm to maximize network lifetime in wireles sensor networks". Journal of Advanced Research in Dynamical and Control Systems, 9(Special Issue 12), 2156-2167.

21. Umar, S., Subbarayudu, Y., Kumar, K. K., \& Bashwanth, N. (2017). calculating Designing of dynamic re-clustering leach protocol for total residual time and performance. International Journal of Electrical and $\begin{array}{lll}\text { Computer } \quad \text { Engineering, } & \text { 7(3), }\end{array}$ doi:10.11591/ijece.v7i3.pp1286-1292

22. Rajendra Prasad, C., \& Bojja, P. (2018). A survey on routing protocols in wireless body area networks for medical applications. Journal of Advanced Research in Dynamical and Control Systems, 10(10 Special Issue), 92-97.

23. Rajendra Dhage, M., \& Vemuru, S. (2018).. Paper presented at the 2017 International Conference on Computing, Communication, "Structural health monitoring of railway tracks using WSN Control and Automation", ICCUBEA 2017, doi:10.1109/ICCUBEA.2017.8463976

24. Regula, T., \& Hussain, M. A. (2018). "Multi-level structured tree based routing for energy efficiency in WSN". International Journal of Engineering and Technology(UAE), 7(2), 5-9. doi:10.14419/ijet.v7i2.32.13515

25. Santhi Vandana, T., \& Sreenivasa Ravi, K. (2018). A survey overview: On wireless body area network and its various applications. International Journal of Engineering and Technology(UAE), 7, 936-940

\section{AUTHORS PROFILE}

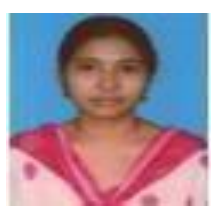

Sk.Shama, working as Assistant Professor of Computer Science and Engineering Department at the Koneru Lakshmaiah Education Foundation situated at Vaddeswarm, received Bachelors Degree from JNTUK, PG from Acharya Nagarjuna University.

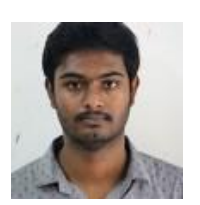

Yashwanth Inuganti, Student, CSE department, Bachelor of Technology, Koneru Lakshmiah Educational Foundation, Andhra Pradesh, India

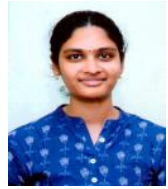

M.B.Bhavana, Student, CSE department, Koneru Lakshmiah Educational Foundation, Andhra Pradesh, India.

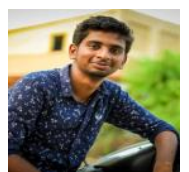

A.Jagapathi, Student,CSE department Bachelor of Technology, Koneru Lakshmiah Educational Foundation, Andhra Pradesh, India

Published By: 